\title{
Design and Implementation of Wireless Sensor Cellular Network Based on Android Platform
}

\author{
https://doi.org/10.3991/ijoe.v13i05.7048 \\ Yan Chen \\ Suzhou Institute of Trade \&Commerce, Suzhou Jiangsu, China \\ zvpbo6214@126.com
}

\begin{abstract}
With the progress of the times, the network has grown into the people's work and life. The limitations of the traditional wireless sensor network (WSN) have become increasingly prominent, and it is obviously unable to meet the requirements of Internet access. Therefore, the 4G mobile Android system is used as a carrier, to design an application layer gateway system for realizing wireless communication between wireless sensor networks and heterogeneous cellular networks, so as to build wireless sensor cellular network. The design and implementation of the AP module, data receiving and forwarding module, network packet loss management and retransmission of the gateway systemis the focus, and then the implemented gateway system is tested. The test results show that the design of the Android platform wireless sensor network gateway system can connect the sensor network to the cellular network, so as to realize data communication, and to ensure that the data loss rate is less than $0.1 \%$. Through testing, it is concluded that the system can meet the actual demand.
\end{abstract}

Keywords-Android system, cellular network, wireless sensor

\section{Introduction}

The Internet of things mainly through the edge of the various access to the perception of the physical world of information to connect to the information world, the two become a unified body [2]. In this process, the perception of the network perception is extremely important. However, from the current reality of many of the specific applications of the Internet industry as well as the current understanding of the industry can be seen, the wireless sensor network (WSN) is an important means to realize the physical perception of the Internet of Things [4]. Internet of Things requires access to any place at any time, and the traditional wireless sensor network is mainly through its sink node to connect with a server in the wired IP network, thus access to the Internet [6]. This is very limited, and it is obviously unable to meet the access requirements of the Internet of things.

In view of the limitations of traditional WSN network and the access requirements of Internet of Things, this paper takes the edge sensor network networking that can access up to Internet whenever and wherever possible as the goal, and consider using 
the existing cellular network system as the intermediary, to realize wireless sensor networks with heterogeneous wireless communication with each other. In this paper, we used the 4G mobile Android system as a carrier, designed an application layer gateway system to realize wireless communication between wireless sensor networks and heterogeneous cellular networks, so as to build wireless sensor cellular network. The mobile phone communicates with the sink node of the wireless sensor network through WiFi technology, and the other side is connected with the Internet via GPRS network. Mobile communication cellular network with high quality coverage, it is basically the global coverage [8], and the mobile communication cellular network has been through GPRS, 4G, LTE and other access network technology and the Internet to achieve a perfect interconnection, using the mobile communication cellular network as an information channel, and the wireless sensor network can also be connected to Internet anytime and anywhere.

\section{State of the art}

Android is a mobile operating platform based on Linux, and the system architecture consists of five parts, they are Linux Kernel, Android Runtime, Libraries, Application Framework and Applications [1]. It is a highly open operating system, with its rapid development and maturity, researchers of Android application development are no longer limited to only use a single Java language [3]. Today's developers can use a variety of development languages to develop colorful and exciting chic android applications, and it has become a true sense of the open operating system [5]. In this study, we mainly use the Android phone as the design of the gateway system carrier.

Through wireless communication, the sensor nodes deployed in a specific area form a self-organizing multi-hop network system, which is a wireless sensor network. The purpose is to realize the sensing, acquisition and processing of the information of the peripheral perceptual objects through the sensor nodes, and send them to a higher level of data processing in the application, such as a server connected by a wired IP. According to this definition, the sensor node, the sensing object and the data processor are the basic elements in the WSN. WSN is the result of cross-cutting between emerging disciplines and traditional disciplines. As a new direction in the field of information technology, wireless sensor has experienced three stages: intelligent sensor, wireless intelligent sensor and wireless sensor network. Intelligent sensor is embedded in the sensor computing power, which makes the sensor node in addition to the basic data acquisition capabilities, but also has the ability of filtering and information processing. On the basis of the intelligent sensor, wireless communication capability is added to form a wireless intelligent sensor, which can extend the perception of the antenna, and can also reduce the cost of the project implementation. Wireless sensor networks are the introduction of network technology in wireless smart sensors, which makes the sensors become an organic combination that can exchange information and coordinate control. Through the infinite extension of the tentacles, we realize the interconnection of things, and it becomes an important component of the Internet of Things. 
Cellular network communication is derived from the characteristics and development of cellular networks, which divides the service of the mobile phone into a subarea of a hexagonal built-in base station. Because it looks like a "honeycomb" structure, we call it cellular mobile communication. The cellular network is mainly composed of three parts: mobile station, base station subsystem and network subsystem. A mobile station or a mobile terminal is our network terminal device, such as a mobile phone. The base station subsystem includes a mobile base station, a wireless transceiver device, a private network, and numerous digital devices. The base station subsystem can be viewed as a converter between a wireless network and a wired network.

At present, the common types of cellular networks are GSM network, CDMA network, FDMA, TDMA, PDC and so on. Cellular networks have gone through four stages, they are analog cellular mobile communications, mobile communications (GSM), 2.5G (GPRS) and 4G. During this period, the confidentiality, spectrum utilization, business richness and standardization of the system are improved. Especially with the rapid development of the communication technology, the study of the cellular wireless location technology is constantly perfected.

The convergence of sensor networks and cellular networks is one of the mainstream in the industry [7]. At present, it is still in the research stage and has not yet been implemented on a large scale. For the integration of the two networks between the strategy, the industry and the academic community of many researchers have given a variety of solutions. It is generally divided into three types: Gateway policies, overlay policies, and wireless mesh policies. Their starting points are different, and the gateway strategy is to provide support for the interconnection of WSN and CN networks by focusing on the information on the application layer. Coverage strategy is mainly focused on the transformation of the protocol stack, and wireless mesh network strategy is to take full advantage of its own agreement with the powerful mobile Internet support function, so as to provide a good way to access.

In summary, the use of mobile communication network to achieve the interconnection of wireless sensor networks and Internet is the consensus of the industry. The integration of mobile communication network and wireless sensor network has also become one of the key projects in the field of research and industrial practice. However, the current situation of the integration of the two networks is still in the stage of research and exploration. Many of the technical standards are still in the development phase of testing. There are no uniform open interface standards. Therefore, in this paper, the $4 \mathrm{G}$ mobile Android system is used as a carrier, to design an application layer gateway system for realizing wireless communication between wireless sensor networks and heterogeneous cellular networks, so as to build wireless sensor cellular network. The design and implementation of the AP module, data receiving and forwarding module, network packet loss management and retransmission of the gateway are the focus, and then the implemented gateway system is tested. 


\section{Design and implementation of wireless sensor cellular network gateway system on Android platform}

The design of the gateway system is to achieve the communication between heterogeneous wireless sensor networks and mobile communication cellular networks. The mobile phone serves as the carrier of the gateway, one side communicates with the sink node of the wireless sensor network, and the other side needs to communicate with the mobile communication cellular network. Considering today's near-field communication (NFC) mode, communication with sink nodes on the wireless sensor network can be achieved using WiFi or Bluetooth and other communication methods. Taking into account the distance between the transmission distance, the system uses WiFi mode. The function of the gateway is to convert the data packets received from the WSN side to the message that can be identified by the mobile communication network, so that it can be sent to the Internet server through the mobile communication network. In view of the fact that API can temporarily not provide message to the mobile communication cellular side in the application layer of the Android package SDK API document, the encapsulation function of mobile communication cellular network side message will be automatically completed by the mobile phone at the bottom of the system.

\subsection{System overall structure design}

The Internet of things gateway combines a variety of functions. The communication protocol and the data types used in the devices of the edge perception layer of the Internet of things are different. It must provide a unified data format conversion and transmission, so as to realize the Internet of things data collection / delivery and transmission. The Internet of things supports a variety of communication protocols and data types of the sensing layer devices. Through the conversion of a variety of information perception layer unified format, and with the integration of network communication gateway function rich, to simplify the design of The Internet of things data collection and transmission system, and provide good maintainability. This system is mainly based on a series of draft issued by the ETSI M2M group, the M2M (Machine to reference Machine) the overall framework and the specific functional requirements of the service application, combined with the basic definition of networking gateway, and reference the "Tan Lu things to explore a beehive" network and wireless sensor network integration in the Internet of things gateway function definition and implementation, to design the gateway function in the gateway system. The system work flow is shown in Figure 1. 


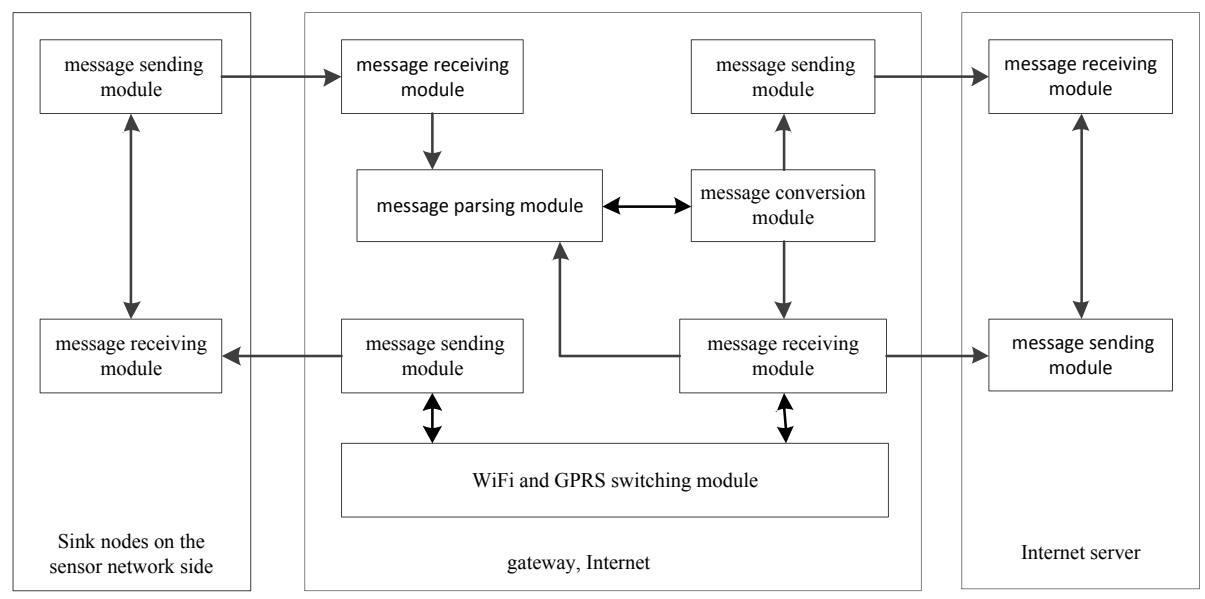

Fig. 1. System work flow chart

\subsection{System topology design}

The network topology can accurately reflect the current structure of the network, and it is very important for the analysis, research and management of wireless sensor networks. Therefore, topology visualization is a very effective and important method to analyze its network structure and node information. However, due to the practical application of the size of the wireless sensor network, and the interaction between nodes is complex and diverse, it is not easy to draw a clear, complete and beautiful network. Combined with the characteristics of the Android platform, we designed the Android platform for wireless sensor cellular network topology. As shown in Figure 2.

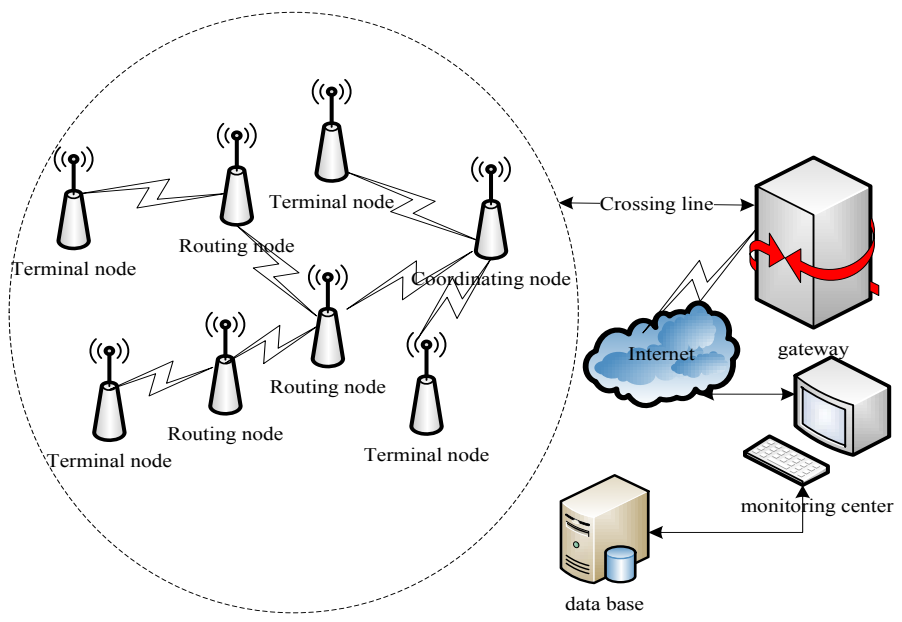

Fig. 2. System work flow chart 
Among them, the gateway is the core of the system, and it connects the sensor network and gateway system. The routing node and the terminal node transmit the collected information to the coordinating node through single hop and multi hop. The coordinating node sends the information to the gateway through the serial line, and the gateway then forwards the information to the monitoring center. Through the monitoring center's friendly software platform, users can remotely monitor and manage wireless sensor networks.

\subsection{Design and implementation of main function module}

According to the system definition described earlier, the system is divided into three parts. The following describes each part of the functional modules.

1) Design of function module on sink node side

Due to the limitations of hardware facilities and other reasons, the system did not really build a large wireless sensor network, so there is no real sense of the sink node. In this system, through the laptop and other mobile phones to simulate the sensor network sink node. In this paper, the function of the sink node is only related to the communication of the system, and the basic function of the sink node in the sensor network is not described here. In this system, a laptop or Android phone simulation of the sink node only has a simple ability to receive data from the gateway and send data to the gateway function.

2) Design of gateway function module

The original intention of this paper is to realize the communication between the terminal and the traditional Internet node. According to this, a very important function of the gateway is to regard itself as "AP (Access Point)". This is equivalent to the gateway to build a wireless communication LAN, which can be achieved in some very remote places and no traditional wireless AP case, through the gateway "AP" and GPRS network to communicate with the server on the Internet. This system uses Android operating system as the carrier of the gateway system, which has the function of WiFi. The basic idea is to make mobile phone running the gateway system in WiFi AP mode, which mainly includes AP module, data receiving and forwarding, packet loss and retransmission, terminal fault management, network congestion control and so on.

Among them, the AP module is mainly responsible for develop the Android phone into AP. At present, either $3 \mathrm{G}$ or $4 \mathrm{G}$ mobile phones have emerged on a lot of mobile phones that can become AP software. The system is used MotoloraME525 + mobile phone comes with the name of Mobile Hotspot software, it can be mobile phone WiFi communication mode in AP mode, and build a mobile phone as the default gateway for a wireless LAN. In this way, the WiFi-enabled terminal device in the area where the handset WiFi signal arrives can be accessed into the local area network, and thus realizing the access function of the application gateway system.

The module of data receiving and forwarding is mainly responsible for receiving and sending data from the gateway side, which includes receiving data from the sensor network, forwarding data to the sink node in the sensor network, forwarding data 
to the server on the Internet, and receiving data from the Internet. The main flow chart of this part is shown in Figure 2.

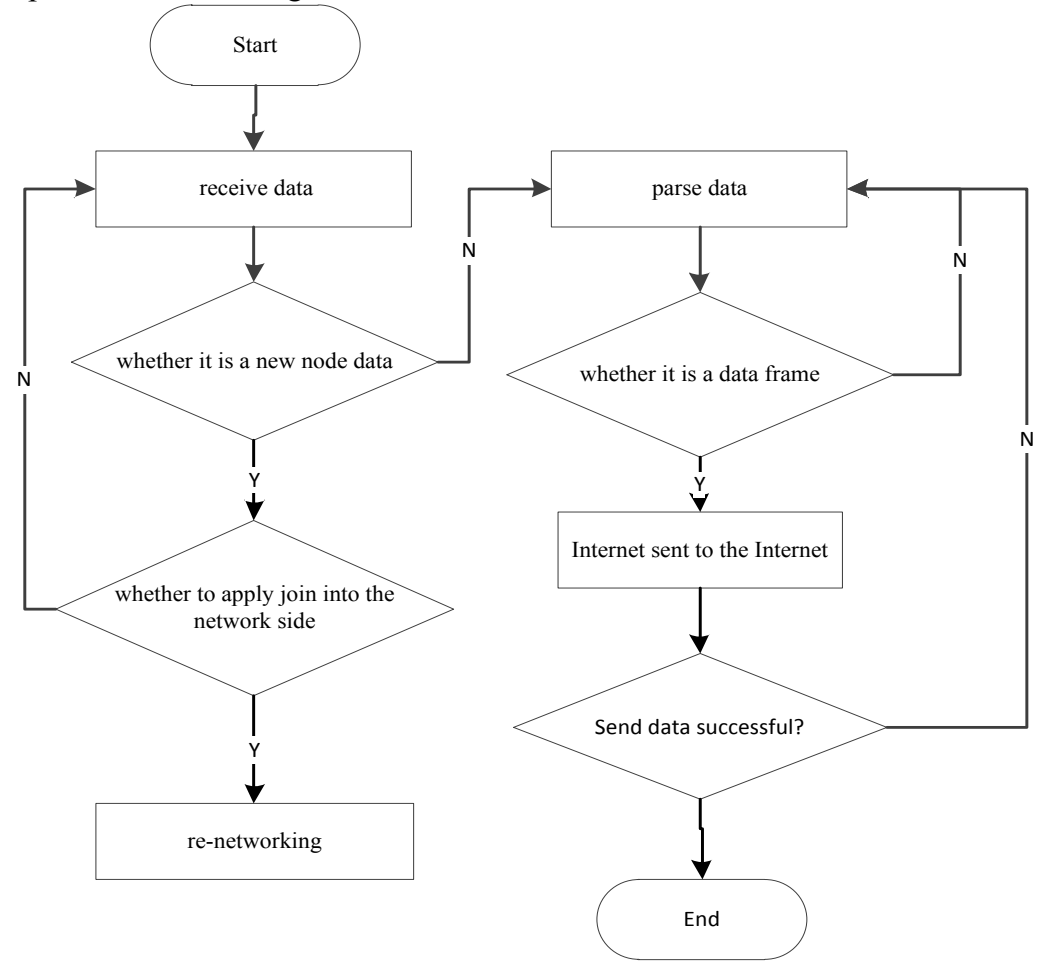

Fig. 3. Data receiving and forwarding flow chart

Network packet loss management and retransmission mechanism is also concerned about the wireless sensor network between the nodes and the wireless communication between the gateways. The retransmission mechanism in this system is: The transmission of the message is synchronous, and the sending direction sends a message to the receiver, the receiver will send back a corresponding ACK message to the sender when it is received correctly. That is, under normal circumstances, after the sender sends the data packet, if the corresponding ACK is not received within time $\mathrm{T}$, the packet will be retransmitted, but the maximum number of retransmissions is $\mathrm{C}$ (the value of $\mathrm{C}$ can be set according to the specific application of the specific network environment). If the sender retransmits $\mathrm{C}$ still does not receive the corresponding ACK, the system will abandon the retransmission mechanism, while disconnect the connection.

Terminal failure management refers to the gateway manages the faults that are likely to be caused by the terminal. The fault management scheme of the gateway terminal given in this system is: The gateway checks the message information sent by the sink node periodically. If a terminal node has no data sent to the gateway within the time range specified by the system, the gateway considers the terminal to be in a 
temporary fault condition. The gateway counts the case where the terminal is in a temporary fault condition. When the count reaches the critical value set by the system, the gateway determines that the terminal is in a faulty state and will process it. If the terminal starts sending data at a certain time before the number of consecutive temporary fault status counts reaches the critical value, then, the gateway determines that the terminal is temporarily faulty and clears the count of the critical state times and restarts such a process. WSN gateway to the terminal fault management process is as shown in Figure 3.

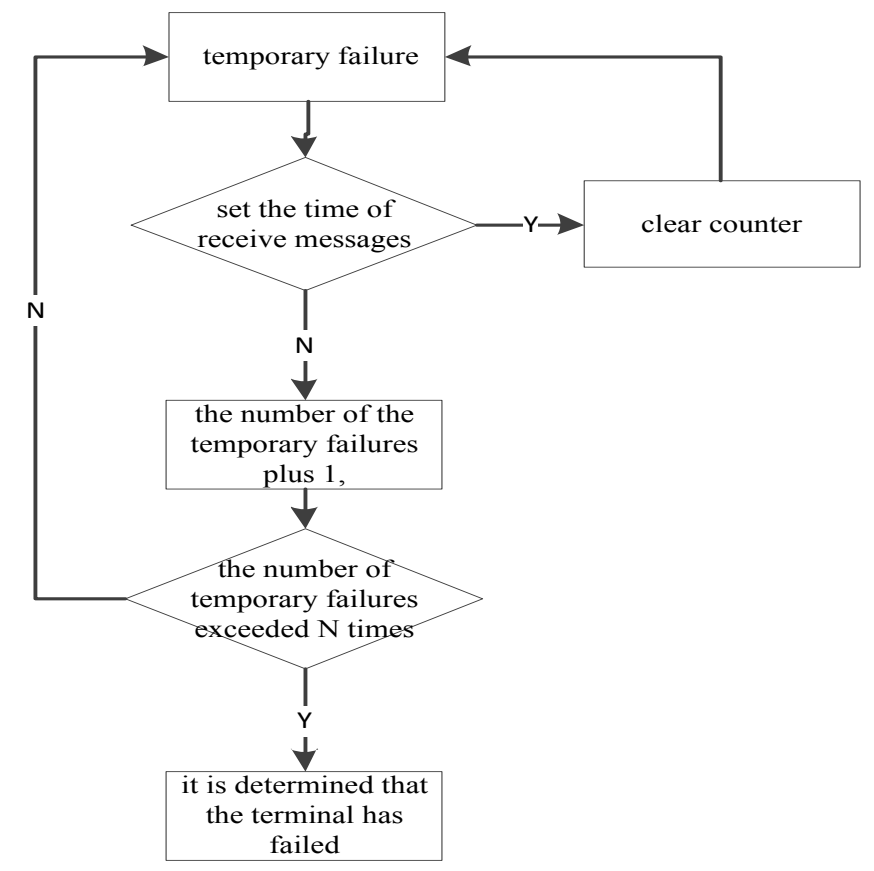

Fig. 4. Terminal fault management flow chart

The control of congestion on the network is a very complex process that involves a multifaceted work. It specifically includes the message processing priority, to record the message queue in the case of packet loss and network congestion after the mitigation and other issues. If we want to do a very good effect of congestion control, it often requires the cooperation of a lot of functional modules. The congestion policy in this system adopts the following priority policy of congestion control strategy, that is to say, when the network congestion occurs, the gateway selects the highest priority message in the current buffer to process the message and discards all the old messages. The priority of the system is divided into eleven levels, from 1 to 10 between the integers, the larger the number, the higher the priority. The priority is carried by the second byte of the baud reserved field.

3) The function of the network server 
Internet server function is relatively simple, that is, communicated with the gateway of a single point, it mainly receives and sends data.

\section{$4 \quad$ Test and result analysis}

The system is an application layer of the gateway, which is mainly used to simulate the legendary side of the notebook computer sink node. One Android phone is as the carrier of the gateway system, another is connected to the Internet pc machine as the system's Internet server. In this paper, we give the main function of the gateway system test (take the packet loss and retransmission function as an example) and performance test results.

(1) Gateway communication distance test

The sensor node sends the collected data to the gateway, by changing the communication distance between the gateway and the node, and through the host computer online debugging software to determine the real-time communication between the gateway and the node, in order to determine the node and the gateway can achieve normal communication between the limit positions. During the test, the mobile intelligent Android mobile phone (Gateway carrier) to change the distance between the gateway and the node, to keep the number of packets sent is unchanged. The experimental results are shown in Figure 4.

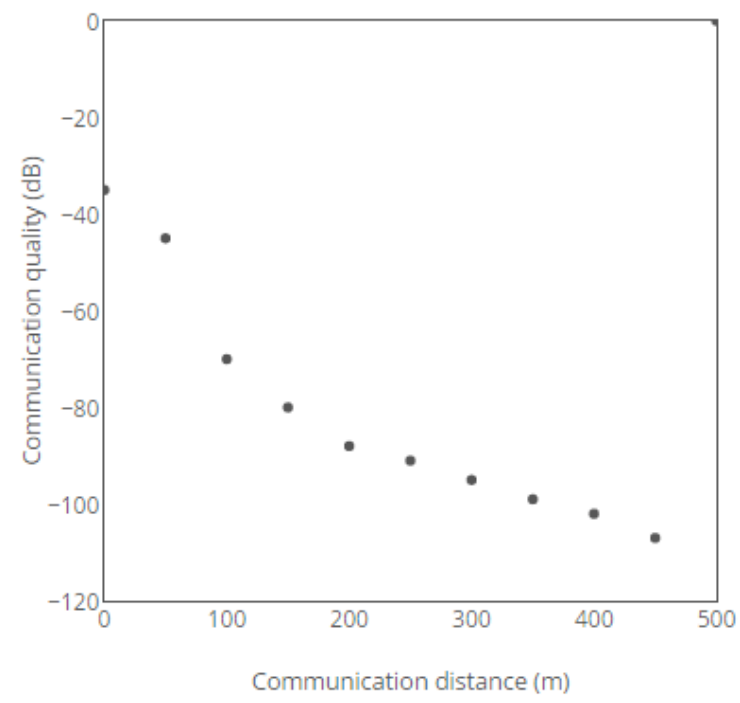

Fig. 5. Communication distance test

When the communication distance is $500 \mathrm{~m}$, the data is empty, indicating that the communication between the gateway and the node is interrupted. As can be seen from Figure 6, when the distance between the gateway and the node is within $450 \mathrm{~m}$, it can keep the communication, once more than this distance, the communication is inter- 
rupted. However, when the distance between the gateway and the node is $350 \mathrm{~m}$ $450 \mathrm{~m}$, although the communication between the two is still able to maintain, but the data transceiver capacity has declined, the quality of communication is about $-100 \mathrm{~dB}$, which can easily lead to data loss. Therefore, in the practical application process, the communication distance between the gateway and the node should be controlled within $300 \mathrm{~m}$.

(2) Data loss rate test

In the test, we gradually increase the number of node unit box, test under different number of nodes, the data loss rate between the gateway and the node, and calculate the average value of packet loss for 5 times. In addition, through the access server is unified timing to all nodes to ensure that the time is synchronization, and adopts the time interval method of timing acquisition and the same acquisition make the nodes collect and transmit the data synchronously. The test results are shown in Table 1.

Table 1. Data packet loss rate statistics

\begin{tabular}{|c|c|}
\hline Number of nodes & Packet loss rate (\%) \\
\hline 1 & 0.007 \\
\hline 2 & 0.01 \\
\hline 4 & 0.02 \\
\hline 6 & 0.03 \\
\hline 8 & 0.05 \\
\hline 10 & 0.09 \\
\hline
\end{tabular}

As can be seen from Table 1, in the data transmission process, when the number of packets remains unchanged, the data loss rate increases gradually with the increase of the number of nodes.

\section{Conclusion}

Based on the existing cellular network system, this paper integrates the wireless sensor network with the cellular network, and realizes the application layer gateway system which can make the edge sensor network of the Internet of Things connect to the Internet anytime and anywhere. The gateway system has some simple management functions and basic data transmission and forwarding functions to the terminal. At the same time, this paper tests the design of the gateway system, the results show that the design of the Android platform of the wireless sensor network gateway system can connect the sensor network and the cellular network, constitute a wireless sensor cellular network and achieve data communication. At the same time, the communication quality of the gateway system is good when the communication distance is within $300 \mathrm{~m}$, it meets the actual needs. When 10 sensor nodes transmit data at the same time, the data loss rate can be controlled within $0.1 \%$, which has good control effect and satisfies the practical requirement. On these bases, we obtain a good experimental result, and achieve the expected goal in real application. 


\section{References}

[1] Han, Q., Liang, S., \& Zhang, H. (2015). Mobile cloud sensing, big data, and 5G networks make an intelligent and smart world [J]. IEEE Network, 29(2), 40-45. https://doi.org/10.1109/MNET.2015.7064901

[2] Jung, S. J., Myllylä, R., \& Chung, W. Y. (2013). Wireless machine-to-machine healthcare solution using android mobile devices in global networks. IEEE Sensors Journal, 13(5), 1419-1424. https://doi.org/10.1109/JSEN.2012.2236013

[3] Lim, W., Torres, H. K., \& Oppus, C. M. (2014, November). An agricultural telemetry system implemented using an Arduino-Android interface. In Humanoid, Nanotechnology, Information Technology, Communication and Control, Environment and Management (HNICEM), 2014 International Conference on (pp. 1-6). IEEE.

[4] Nandkishor, B. R., Shinde, A., \& Malathi, P. (2014, August). Android smartphone based body area network for monitoring and evaluation of medical parameters. In Networks \& Soft Computing (ICNSC), 2014 First International Conference on (pp. 284-288). IEEE. https://doi.org/10.1109/cnsc.2014.6906663

[5] Piyare, R. (2013). Internet of things: ubiquitous home control and monitoring system using android based smart phone. International Journal of Internet of Things, 2(1), 5-11.

[6] Rawat, P., Singh, K. D., Chaouchi, H., \& Bonnin, J. M. (2014). Wireless sensor networks: a survey on recent developments and potential synergies. The Journal of supercomputing, 68(1), 1-48. https://doi.org/10.1007/s11227-013-1021-9

[7] Saranya, J., \& Selvakumar, J. (2013, April). Implementation of children tracking system on android mobile terminals. In Communications and Signal Processing (ICCSP), 2013 International Conference on (pp. 961-965). IEEE. https://doi.org/10.1109/iccsp.2013.65771 99

[8] Shivakumar, N. S., \& Sasikala, M. (2014, March). Design of vital sign monitor based on wireless sensor networks and telemedicine technology. In Green Computing Communication and Electrical Engineering (ICGCCEE), 2014 International Conference on (pp. 1-5). IEEE. https://doi.org/10.1109/icgccee.2014.6922257

\section{$7 \quad$ Author}

Yan Chen is Associate Professor at the Institute of Electrical and Information Technology, Suzhou Institute of Trade \&Commerce, Suzhou Jiangsu 215009, China (zvpbo6214@126.com). 